\title{
HETEROSIS AND COMBINING ABILITY IN DIALLEL CROSSES AMONG CULTIVARS OF UPLAND COTTON
}

(Received: 7.6.2009)

\author{
By \\ Kh. M. Dawod and Kh. Kh. Al-Guboory* \\ College of Agriculture \& Forestry, Mosul University and ${ }^{*}$ College of Agriculture, Karkuk University, Iraq
}

\begin{abstract}
A seven parent (Halab $90=\mathrm{P}_{1}$, SP $8886=\mathrm{P}_{2}$, Dunn $1517=\mathrm{P}_{3}$, Coker $5114=\mathrm{P}_{4}$, Lachata $=\mathrm{P}_{5}$, Dunn $1047=\mathrm{P}_{6}$ and Coker $310=\mathrm{P}_{7}$ ) diallel of upland cotton was planted in a randomized complete block design with three replications at Al-Haweja, Karkuk Governorate during the season of 2007. Data collected from the diallel were used to estimate combining ability and heterosis for plant height, number of bolls per plant, boll weight, lint index, ginning outturn and seed cotton yield. The results showed that the hybrids $\mathrm{P}_{1} \times \mathrm{P}_{4}, \mathrm{P}_{3} \times \mathrm{P}_{5}, \mathrm{P}_{4} \times \mathrm{P}_{5}, \mathrm{P}_{5} \times \mathrm{P}_{6}$ and $\mathrm{P}_{5} \times \mathrm{P}_{7}$ had significantly positive mid parents and higher parent heterosis for a larger number of characters. Significant variances of genotype, parents vs crosses, general combining ability (gca) and specific combining ability (sca) were observed for all studied characters. Almost additive genetic variance was preponderant for plant height, boll weight, ginning outturn and seed cotton yield and non-additive gene action was involved in the number of bolls per plant and lint index. The two cultivars Coker 310 and Lachata, and the crosses $\mathrm{P}_{1} \times \mathrm{P}_{4}$ and $\mathrm{P}_{5} \times \mathrm{P}_{7}$ exhibited significant positive gca and sca effects respectively for a larger number of characters, and were found to be the best general and specific combiners, and could be used for future breeding programmes.
\end{abstract}

Key words: genotypes, heterosis, combining ability, upland cotton.

\section{INTRODUCTION}

Cotton as a commercial crop has played an important role in boosting national economy of several countries, and provides fiber, food, feed and fuel to high percentage of people as well as livestock (Ahmad et al., 2005). The increase in yield per unit area of the crop is a prime concern of breeding programmes, and cotton breeders all over the world. They have been utilizing genetic resources to modify the cultivars to meet the ever changing requirements of their society. The first step in a successful breeding programme is to select appropriate parents. Diallel analysis provides a systematic approach for the detection of appropriate parents and crosses superior in terms of the investigated traits (Basal and Turgut, 2003). It also helps plant breeders to choose the most efficient selection method by allowing them to estimate several genetic parameters (Verhalen and Murray, 1967).

Heterosis is the superiority in performance of hybrid individuals compared with their parents. Regarding previous studies on heterosis in cotton, Salam (1991), Altaf et al. (1996) and Abro et al. (2009) conducted such studies for yield contributing characters and reported promising heterosis for yield parameters, and through heterosis, seed cotton contributing characters can be improved significantly (Naquibullah et al., 2000).

Combining ability describes the breeding value of parental lines to produce hybrids. Sprague and Tatum (1942) used the term general combining ability (gca) to designate the average performance of a line in hybrid combinations, and used the term specific combining ability (sca) to define those cases in which certain combinations do relatively better or worse than the expected on the basis of the average performance of the lines involved.

In order to choose appropriate parents and crosses, and to determine the combining abilities of parents in the early generations, the diallel analysis method has been widely used by plant breeders. This method was applied to improve self and cross-pollinated plants (Jinks and Hayman, 1953; Hayman, 1954; Jinks, 1956; Griffing, 1956; and Hayman, 1960). The importance of combining ability studies lies in the assessment of of parental lines and their 
hybrids showing significant additive and non additive effect with respect to certain characters. In a systematic breeding programme, it is essential to identify superior parents for hybridization and crosses to expand the genetic variability for selection of superior genotypes (Inamullah et al. 2006).

The present study with $7 \times 7$ diallel cross was undertaken for isolating superior cultivars and better combining parents for suitable hybrids.

\section{MATERIALS AND METHODS}

Seven cultivars of upland cotton (Halab $90=$ $\mathrm{P}_{1}$, SP $8886=\mathrm{P}_{2}$, Dunn $1517=\mathrm{P}_{3}$, Coker $5114=$ $\mathrm{P}_{4}$, Lachata $=\mathrm{P}_{5}$, Dunn $1047=\mathrm{P}_{6}$ and Coker 310 $=\mathrm{P}_{7}$ ) were mated in a diallel fashion excluding the reciprocals during the season of 2006. The resulting $21 \mathrm{~F}_{1} \mathrm{~s}$ and their parents were planted at Al-Haweja, Karkuk Governorate, Iraq, in mid April, 2007 using a randomized complete block design with three replications. Each plot consisted of three rows of $5 \mathrm{~m}$ length. The spacing between rows was $75 \mathrm{~cm}$ and plant to plant was $25 \mathrm{~cm}$. One plant per hill was maintained. Fertilizers were applied at the rate of $200 \mathrm{~kg} /$ hectare $\mathrm{P}_{2} \mathrm{O}_{5}$ before planting, and 200 $\mathrm{kg} /$ hectare $\mathrm{N}$ as urea at twice, the first time after germination and the second at the beginning of flowering.

Observations were recorded on ten randomly selected guarded plants from each plot for plant height $(\mathrm{cm})$, number of bolls per plant, boll weight (gm), lint index (gm fiber per 100 seeds), ginning outturn (ratio of fiber cotton weight in the sample to the seed cotton yield as percentage) and seed cotton yield per plant (gm).

Data of the genotypes (parents and their $\mathrm{F}_{1} \mathrm{~s}$ ) were subjected to analysis of variance for all the characters studied according to the method of experimental design used, and comparisons between means were done according to Duncan's Multiple Range Test method (Steel and Torrie, 1980). General combining ability (gca) and specific combining ability (sca) effects were estimated by following Model -1 , method 2 of Griffing (1956). The mean squares for gca and sca were tested against error variance desired. Mid parent (MP) and higher parent (HP) heterosis (Halluer and Miranda, 1981) values were calculated by using the means of the parents and $\mathrm{F}_{1} \mathrm{~s}$ and through the formulas: $\left(\mathrm{F}_{1}-\right.$ $\mathrm{MP}) / \mathrm{MP}$ and $\left(\mathrm{F}_{1}-\mathrm{HP}\right) / \mathrm{HP}$, respectively. All statistical analyses were performed by using SAS (Statistical Analyses System V. 9) and Microsoft Office Excel 2003.

\section{RESULTS AND DISCUSSION}

Mid parent heterosis and higher parent heterosis for different characters in cotton are presented in Table (1). For plant height, the cross $\begin{array}{llllll}\mathrm{P}_{1} & \mathrm{x} & \mathrm{P}_{6} & \text { has the highest positive (desirable) }\end{array}$ heterosis over the both higher parent and mid parents. Heterosis over mid parent was found in the range of $-15.4 \%$ to $79 \%$. The crosses $\mathrm{P}_{2} \times \mathrm{P}_{4}$, $\mathrm{P}_{3} \times \mathrm{P}_{4}$ and $\mathrm{P}_{4} \times \mathrm{P}_{7}$ showed significant and negative (undesirable) heterosis over the higher parent as well as mid parents, while the estimates of heterosis were significantly positive in fourteen of the crosses. Heterosis over higher parent for the numbers of bolls per plant ranged from $-33.4 \%$ to $48.3 \%$. Significant positive heterosis over both higher parent and mid parents was observed in crosses $\mathrm{P}_{1} \times \mathrm{P}_{6}, \mathrm{P}_{1} \times \mathrm{P}_{7}, \mathrm{P}_{4} \times \mathrm{P}_{5}$, $\mathrm{P}_{4} \times \mathrm{P}_{6}, \mathrm{P}_{4} \times \mathrm{P}_{7}, \mathrm{P}_{5} \times \mathrm{P}_{6}, \mathrm{P}_{5} \times \mathrm{P}_{7}$ and $\mathrm{P}_{6} \times \mathrm{P}_{7}$, with highest percents in the cross $\mathrm{P}_{4} \times \mathrm{P}_{5}$. Nine crosses showed significantly negative heterosis over both higher parent and mid parents. For boll weight, significant positive heterosis was observed in the crosses $\mathrm{P}_{1} \times \mathrm{P}_{3}, \mathrm{P}_{1} \times \mathrm{P}_{5}, \mathrm{P}_{2} \times \mathrm{P}_{4}$ and $\mathrm{P}_{3} \times \mathrm{P}_{5}$ over higher parent as well as mid parents, while the crosses $\mathrm{P}_{3} \times \mathrm{P}_{6}, \mathrm{P}_{4} \times \mathrm{P}_{6}$ and $\mathrm{P}_{6} \times \mathrm{P}_{7}$ showed significant negative heterosis. Heterosis of lint index over higher parent and mid parents varied from $-25.2 \%$ to $13.6 \%$ and -24.9 to $8.16 \%$ respectively. The cross $\mathrm{P}_{1} \quad \mathrm{x} \quad \mathrm{P}_{6}$ showed significant positive heterosis over higher parent and mid parents, while significantly negative heterosis was observed in fourteen other crosses over higher parent and mid parents for this character. The highest heterosis percents over higher parent and mid parents were exhibited by the crosses $\mathrm{P}_{2} \times \mathrm{P}_{7}$ and $\mathrm{P}_{3} \times \mathrm{P}_{4}$ respectively. For ginning outturn, the crosses $\mathrm{P}_{1} \times \mathrm{P}_{4}, \mathrm{P}_{1} \times \mathrm{P}_{7}, \mathrm{P}_{2} \times$ $\mathrm{P}_{4}, \mathrm{P}_{2} \times \mathrm{P}_{5}, \mathrm{P}_{3} \times \mathrm{P}_{6}, \mathrm{P}_{3} \times \mathrm{P}_{7}, \mathrm{P}_{4} \times \mathrm{P}_{5}, \mathrm{P}_{4} \times \mathrm{P}_{6}, \mathrm{P}_{5} \times$ $\mathrm{P}_{6}$ and $\mathrm{P}_{5} \times \mathrm{P}_{7}$ showed significant positive heterosis, and the crosses $\mathrm{P}_{1} \times \mathrm{P}_{2}, \mathrm{P}_{1} \times \mathrm{P}_{5}, \mathrm{P}_{1} \times \mathrm{P}_{6}$ and $\mathrm{P}_{2} \times \mathrm{P}_{7}$ showed significant negative heterosis over higher and mid parents. The highest percentages of heterosis over mid parents and higher parent were exhibited by the crosses $\mathrm{P}_{5} \mathrm{X}$ $\mathrm{P}_{6}$ and $\mathrm{P}_{4} \times \mathrm{P}_{5}$ respectively. The crosses $\mathrm{P}_{1} \times \mathrm{P}_{4}$, $\mathrm{P}_{1} \times \mathrm{P}_{5}, \mathrm{P}_{2} \times \mathrm{P}_{7}, \mathrm{P}_{3} \times \mathrm{P}_{5}, \mathrm{P}_{4} \times \mathrm{P}_{5}, \mathrm{P}_{5} \times \mathrm{P}_{6}$ and $\mathrm{P}_{5} \times$ $\mathrm{P}_{7}$ showed significant and positive heterosis for seed cotton yield per plant, over better parent and mid parents, while significantly negative heterosis was exhibited by the crosses $\mathrm{P}_{1} \times \mathrm{P}_{3}, \mathrm{P}_{2}$ $x \mathrm{P}_{3}$ and $\mathrm{P}_{3} \times \mathrm{P}_{7}$. The cross $\mathrm{P}_{4} \times \mathrm{P}_{5}$ has the highest percent of mid parent heterosis and better parent heterosis. These findings are supported by other researchers like Khan et al. (1999), Mukhtar and Khan (2000), Solangi et al. (2002), Abro et al. 
Table (1): Heterosis over mid parents and higher parent (\%) for different characters for 21 crosses in upland cotton.

\begin{tabular}{|c|c|c|c|c|c|c|c|c|c|c|c|c|}
\hline \multirow{2}{*}{ cross } & \multicolumn{2}{|c|}{ Plant height } & \multicolumn{2}{|c|}{ No. bolls per plant } & \multicolumn{2}{|c|}{ Boll weight } & \multicolumn{2}{|c|}{ Lint index } & \multicolumn{2}{|c|}{ Ginning outturn } & \multicolumn{2}{|c|}{ Seed cotton yield } \\
\hline & MP & HP & MP & HP & MP & HP & MP & HP & MP & HP & MP & HP \\
\hline $\mathbf{P}_{\mathbf{1}} \times \mathbf{P}_{\mathbf{2}}$ & 61.9** & $40.9 * *$ & 0.689 & $-4.84 * *$ & $11.1 * *$ & -0.79 & $3.70 * *$ & -0.59 & $-3.63 * *$ & $-3.77 * *$ & $11.1 * *$ & $-5.59 *$ \\
\hline $\mathbf{P}_{\mathbf{1}} \times \mathbf{P}_{\mathbf{3}}$ & $20.2 * *$ & $-7.2 * *$ & $-25.4 * *$ & $-33.4 * *$ & $27.3 * *$ & 20.9** & $-12.2 * *$ & $-12.7 * *$ & $1.03 * *$ & 0.09 & $-5.65 *$ & $-19.5 * *$ \\
\hline $\mathbf{P}_{1} \times \mathbf{P}_{4}$ & $27.7 * *$ & $-4.2 * *$ & $5.53 * *$ & 1.78 & $15.9 * *$ & 3.15 & $5.47 * *$ & -0.65 & $6.81 * *$ & $2.19 * *$ & $22.9 * *$ & $13.0 * *$ \\
\hline $\mathbf{P}_{1} \times \mathbf{P}_{5}$ & $22.7 * *$ & $4.00 * *$ & $3.50 * *$ & $-2.62 *$ & $18.8 * *$ & $4.62 *$ & $-4.94 * *$ & $-8.88 * *$ & $-0.99 * *$ & $-5.67 * *$ & $24.0 * *$ & $15.5 * *$ \\
\hline $\mathbf{P}_{1} \times \mathbf{P}_{6}$ & 79.0** & 63.9** & $11.58 * *$ & $3.77 * *$ & 1.19 & $-16.9 * *$ & $4.43 * *$ & $2.48 *$ & $-3.26 * *$ & $-5.57 * *$ & $14.8 * *$ & 0.29 \\
\hline $\mathbf{P}_{\mathbf{1}} \times \mathbf{P}_{7}$ & $17.5 * *$ & $-9.4 * *$ & $12.14 * *$ & $11.09 * *$ & $8.24 * *$ & $-11.5 * *$ & $-24.9 * *$ & $-25.2 * *$ & $9.74 * *$ & $8.57 * *$ & $21.7 * *$ & 0.17 \\
\hline $\mathbf{P}_{\mathbf{2}} \times \mathbf{P}_{\mathbf{3}}$ & $30.6 * *$ & $13.3 * *$ & $-28.1 * *$ & $-32.3 * *$ & $5.93 * *$ & -0.79 & $-15.3 * *$ & $-18.3 * *$ & 0.98** & 0.19 & $-23.5 * *$ & $-23.9 * *$ \\
\hline $\mathbf{P}_{2} \times \mathbf{P}_{4}$ & $-5.4 * *$ & $-20.8 * *$ & $\mathbf{- 8 . 4 6} * *$ & $-16.4 * *$ & $13.8 * *$ & $13.4 * *$ & $-15.0 * *$ & $-23.1 * *$ & $7.37 * *$ & $2.58 * *$ & 4.22* & $-4.47 *$ \\
\hline $\mathbf{P}_{2} \times \mathbf{P}_{5}$ & 33.3** & $29.2 * *$ & $-3.39 * *$ & $-13.8 * *$ & 3.13 & 1.54 & $-7.69 * *$ & $-7.69 * *$ & $13.2 * *$ & $7.74 * *$ & -0.11 & $-9.60 * *$ \\
\hline $\mathbf{P}_{\mathbf{2}} \times \mathbf{P}_{6}$ & $48.3 * *$ & $40.3 * *$ & $-5.49 * *$ & $-16.6 * *$ & $3.57 *$ & $-5.84 * *$ & $-7.88 * *$ & $-10.1 * *$ & $2.19 * *$ & -0.39 & -0.81 & -4.01 \\
\hline $\mathbf{P}_{\mathbf{2}} \times \mathbf{P}_{7}$ & $6.28 * *$ & 41.1** & $-4.37 * *$ & $-10.4 * *$ & $12.8 * *$ & 1.92 & $-9.59 * *$ & $13.6 * *$ & $-0.74 * *$ & $-1.66 * *$ & 8.64** & 4.56* \\
\hline $\mathbf{P}_{\mathbf{3}} \times \mathbf{P}_{4}$ & $-15.4 * *$ & $-18.9 * *$ & $-21.6 * *$ & $-32.2 * *$ & $9.71 * *$ & 2.39 & 8.16** & 1.27 & 2.78*** & $-2.54 * *$ & $-13.0 * *$ & -4.92 \\
\hline $\mathbf{P}_{\mathbf{3}} \times \mathbf{P}_{5}$ & $15.9 * *$ & $3.44 * *$ & $11.1 * *$ & $-5.92 * *$ & $20.0 * *$ & $10.8 * *$ & $-17.2 * *$ & $-20.1 * *$ & $4.65 * *$ & $-1.17 * *$ & $35.4 * *$ & $23.1 * *$ \\
\hline $\mathbf{P}_{3} \times \mathbf{P}_{6}$ & $23.7 * *$ & $2.47 * *$ & $-4.51 * *$ & $-19.9 * *$ & $-3.79 *$ & $-17.5 * *$ & $-11.9 * *$ & $-13.1 * *$ & $9.74 * *$ & $6.15 * *$ & $-5.09 *$ & -2.38 \\
\hline $\mathbf{P}_{\mathbf{3}} \times \mathbf{P}_{7}$ & $13.2 * *$ & $12.8 * *$ & $-12.7 * *$ & $-22.7 * *$ & 3.01 & $-12.2 * *$ & 1.61 & 0.64 & $6.19 * *$ & $6.04 * *$ & $\mathbf{- 8 . 0 5} * *$ & $-11.9 * *$ \\
\hline $\mathbf{P}_{4} \times \mathbf{P}_{5}$ & $20.1 * *$ & $3.18 * *$ & $52.2 * *$ & $48.3 * *$ & 1.95 & 0.77 & $-11.1 * *$ & $-19.5 * *$ & $12.4 * *$ & $11.9 * *$ & $55.1 * *$ & 52.9** \\
\hline $\mathbf{P}_{4} \times \mathbf{P}_{6}$ & $47.9 * *$ & $18.5 * *$ & $13.8 * *$ & $9.57 * *$ & $-4.63 *$ & $-12.9 * *$ & $-13.4 * *$ & $-19.9 * *$ & $6.67 * *$ & $4.49 * *$ & $8.92 * *$ & 2.98 \\
\hline $\mathbf{P}_{4} \times \mathbf{P}_{7}$ & $-7.1 * *$ & $-10.7 * *$ & 9.31** & $6.39 * *$ & $7.42 * *$ & -2.56 & -0.34 & $-5.84 * *$ & $2.83 * *$ & $-2.63 * *$ & $17.1 * *$ & 3.69 \\
\hline $\mathbf{P}_{5} \times \mathbf{P}_{6}$ & $48.2 * *$ & $36.1 * *$ & $13.8 * *$ & $12.3 * *$ & $11.9 * *$ & 3.25 & $-13.3 * *$ & $-15.4 * *$ & $13.6^{* * *}$ & $10.8 * *$ & 27.51** & $18.9 * *$ \\
\hline $\mathbf{P}_{5} \times \mathbf{P}_{7}$ & $22.3 * *$ & 8.79** & $28.9 * *$ & $22.4 * *$ & $7.69 * *$ & -1.28 & $-3.41 * *$ & $-7.69 * *$ & $6.87 * *$ & 0.78** & $38.2 * *$ & $20.8 * *$ \\
\hline $\mathbf{P}_{6} \times \mathbf{P}_{7}$ & $12.6 * *$ & $-6.97 * *$ & 23.8** & $16.1 * *$ & $-13.5 * *$ & $-14.1 * *$ & $-7.30 * *$ & $-9.32 * *$ & 4.03** & 0.49 & $6.95 * *$ & -0.26 \\
\hline
\end{tabular}

$(* *)$ and $(*)$ significant at $5 \%$ and $1 \%$ levels respectively.

$P_{1}=$ Halab 90, $P_{2}=$ SP 8886, $P_{3}=$ Dunn 1517, $P_{4}=$ Coker 5114, $P_{5}=$ Lachata, $P_{6}=$ Dunn 1047 and $P_{7}=$ Coker 310

MP = mid parents $\quad \mathrm{HP}=$ higher parent 
(2009) who also reported a fair degrees of heterosis over mid parents and higher parent for different characters. From the previous information it is concluded that the hybrids $\mathrm{P}_{1} \mathrm{x}$ $\mathrm{P}_{4}, \mathrm{P}_{3} \times \mathrm{P}_{5}, \mathrm{P}_{4} \times \mathrm{P}_{5}, \mathrm{P}_{5} \times \mathrm{P}_{6}$ and $\mathrm{P}_{5} \times \mathrm{P}_{7}$ have significantly positive heterosis over mid parents and higher parent for larger number of traits as compared with the other hybrids, and that the cultivar $\mathrm{P}_{5}$ (Lachata) was shared a common factor in four of these hybrids.

Analysis of variance for the genotypes and combining ability (gca and sca) is presented in Table (2). Genotypes and parents vs. crosses mean squares were highly significant for all studied characters which indicate considerable distance among the genotypes and over all parents and crosses means respectively. Mean squares of gca and sca were also highly significant for all characters, which indicates additive and non additive types of the gene action involved in the manifestation of characters under study. These findings are in accordance with those of Zia-ul-Islam et al. (2001), Deshpande and Baig (2003) and Abro et al. (2009). The mean squares due to sca was much higher than gca for the number of bolls per plant and lint index which revealed the predominance of non additive gene action for controlling these characters. The higher magnitude of gca variance was found for boll weight and seed cotton yield which indicated predominance of additive gene action, while both additive and non additive gene action showed similar importance for plant height and ginning outturn. Ahmad et al. (2005) reported similar results for number of bolls per plant. Abro et al. (2009) also reported predominance of additive gene action for seed cotton yield per plant.

Table (2): Mean square due to genotypes, gca and sca for different characters in cotton.

\begin{tabular}{|c|c|c|c|c|c|c|c|}
\hline $\begin{array}{l}\text { Source of } \\
\text { variation }\end{array}$ & d.f. & $\begin{array}{c}\text { Plant } \\
\text { height }(\mathrm{cm})\end{array}$ & $\begin{array}{l}\text { No. bolls } \\
\text { per plant }\end{array}$ & $\begin{array}{c}\text { Boll } \\
\text { weight } \\
\text { (gm) }\end{array}$ & $\begin{array}{l}\text { Lint index } \\
\text { (gm) }\end{array}$ & $\begin{array}{c}\text { Ginning } \\
\text { outturn } \\
(\%)\end{array}$ & $\begin{array}{l}\text { Yield } \\
(\mathrm{gm})\end{array}$ \\
\hline Reps. & 2 & 0.202 & 0.922 & 0.004 & 0.002 & $\mathbf{0 . 0 3 7}$ & 26.06 \\
\hline Genotypes & 27 & 750.9** & $50.41 * *$ & $0.659 * *$ & $0.592 * *$ & 8.046** & $1527.7 * *$ \\
\hline (Parents) & (6) & $1248.02 * *$ & $64.95 * *$ & $1.461 * *$ & $0.398 * *$ & $8.228 * *$ & $1182.2 * *$ \\
\hline (Crosses) & (20) & 369.05** & $48.19 * *$ & $0.379 * *$ & $0.563 * *$ & 6.461** & $1540.2 * *$ \\
\hline (P. vs crosses) & (1) & $5405.9 * *$ & $7.61 * *$ & $1.463 * *$ & $2.343 * *$ & $38.66 * *$ & 3351.1** \\
\hline Error & 54 & 0.105 & 0.286 & 0.017 & 0.007 & 0.016 & 21.284 \\
\hline gca & (6) & $765.5^{* *}$ & $17.97 * *$ & $1.711 * *$ & $0.417 * *$ & $8.718 * *$ & $2698.4 * *$ \\
\hline sca & (21) & 746.8** & $59.68 * *$ & 0.359** & $0.642 * *$ & $7.854 * *$ & $1193.3 * *$ \\
\hline Error & 54 & 0.035 & 0.095 & 0.006 & 0.002 & 0.006 & 7.095 \\
\hline \multicolumn{2}{|c|}{ gca / sca } & 1.024 & 0.301 & 4.766 & 0.649 & 1.110 & 2.261 \\
\hline
\end{tabular}

$(* *)$ and $(*)$ significant at $5 \%$ and $1 \%$ levels respectively.

Table (3): Mean performance and gca effects $\left(g_{i}\right)$ for different characters in cotton.

\begin{tabular}{|c|c|c|c|c|c|c|c|}
\hline Parents & & $\begin{array}{l}\text { Plant height } \\
\text { (cm) }\end{array}$ & $\begin{array}{l}\text { No. bolls per } \\
\text { plant }\end{array}$ & $\begin{array}{l}\text { Boll weight } \\
\text { (gm) }\end{array}$ & $\begin{array}{l}\text { Lint index } \\
\text { (gm) }\end{array}$ & $\begin{array}{c}\text { Ginning } \\
\text { outturn }(\%)\end{array}$ & $\begin{array}{l}\text { Yield } \\
\text { (gm) }\end{array}$ \\
\hline \multirow{2}{*}{$\mathbf{P}_{1}$} & mean & $54.50 \mathrm{f}$ & $31.867 \mathrm{c}$ & $3.300 \mathrm{~d}$ & $5.167 \mathrm{c}$ & $33.50 \mathrm{~b}$ & 105.09 e \\
\hline & $g_{i}$ & $(-9.527) *$ & $(-0.454) *$ & $(-0.358) *$ & $(0.052)^{*}$ & $(-0.242) *$ & $(-13.355)^{*}$ \\
\hline \multirow{2}{*}{$\mathbf{P}_{2}$} & mean & $73.50 \mathrm{~d}$ & $35.800 \mathrm{~b}$ & $4.200 \mathrm{~b}$ & $5.633 \mathrm{a}$ & $33.60 \mathrm{~b}$ & $150.35 \mathrm{~b}$ \\
\hline & $\mathbf{g}_{\mathbf{i}}$ & $(-3.171)^{*}$ & $(-0.554) *$ & $(-0.014)$ & $(0.141) *$ & $(0.135)^{*}$ & $(-3.051)^{*}$ \\
\hline \multirow{2}{*}{$\mathbf{P}_{3}$} & mean & $99.90 \mathrm{~b}$ & $40.533 \mathrm{a}$ & $3.667 \mathrm{c}$ & $5.233 \mathrm{bc}$ & $34.13 \mathrm{a}$ & $148.67 \mathrm{bc}$ \\
\hline & $g_{i}$ & $(4.188)^{*}$ & $(0.453)^{*}$ & $(-0.258)^{*}$ & $(-0.033)^{*}$ & $(0.650)^{*}$ & $(-7.144)^{*}$ \\
\hline \multirow{2}{*}{$\mathbf{P}_{4}$} & mean & 109.0 a & $29.600 \mathrm{~d}$ & $4.233 \mathrm{~b}$ & $4.567 \mathrm{~d}$ & $30.60 \mathrm{~d}$ & $125.30 \mathrm{~d}$ \\
\hline & $g_{i}$ & $(6.722)^{*}$ & $(-0.239) *$ & $(-0.036) *$ & $(-0.233)^{*}$ & $(-0.694) *$ & $(-2.025)^{*}$ \\
\hline \multirow{2}{*}{$\mathbf{P}_{5}$} & mean & 78.33 c & 28.100 e & $4.333 \mathrm{~b}$ & $5.633 \mathrm{a}$ & $30.33 \mathrm{~d}$ & $121.77 \mathrm{~d}$ \\
\hline & $\mathbf{g}_{\mathbf{i}}$ & $(-0.485)^{*}$ & $(1.016) *$ & $(0.112)^{*}$ & $(0.104)^{*}$ & $(-0.449) *$ & $(8.922)^{*}$ \\
\hline \multirow{2}{*}{$\mathbf{P}_{6}$} & mean & $65.57 \mathrm{e}$ & $27.400 \mathrm{e}$ & $5.133 \mathrm{a}$ & $5.367 \mathrm{~b}$ & $31.90 \mathrm{c}$ & $140.65 \mathrm{c}$ \\
\hline & $\mathrm{g}_{\mathrm{i}}$ & $(-0.234) *$ & $(-1.150)^{*}$ & $(0.183)^{*}$ & $(0.026)^{*}$ & $(-0.235)^{*}$ & $(0.003)$ \\
\hline \multirow{2}{*}{$\mathbf{P}_{7}$} & mean & $100.5 \mathrm{~b}$ & 31.267 c & $5.200 \mathrm{a}$ & $5.133 \mathrm{c}$ & $34.23 \mathrm{a}$ & $162.55 \mathrm{a}$ \\
\hline & $\mathbf{g}_{\mathbf{i}}$ & $(2.507)^{*}$ & $(0.928)^{*}$ & $(0.371)^{*}$ & $(-0.056)^{*}$ & $(0.835)^{*}$ & $(16.650)^{*}$ \\
\hline \multicolumn{2}{|c|}{ P. means } & 83.043 & 32.081 & 4.295 & 5.248 & 32.614 & 136.342 \\
\hline \multicolumn{2}{|c|}{ SE (gi) } & 0.088 & 0.146 & 0.035 & 0.023 & 0.035 & 1.256 \\
\hline
\end{tabular}

$P_{1}=$ Halab 90, $P_{2}=S P$ 8886, $P_{3}=$ Dunn 1517, $P_{4}=$ Coker 5114, $P_{5}=$ Lachata, $P_{6}=$ Dunn 1047 and $P_{7}=$ Coker 310 
Means of the studied characters (x) and gca effects $\left(g_{i}\right)$ of the parents are given in Table (3). Duncan multiple range test showed significant differences between parent means for all the studied characters. While $\mathrm{P}_{7}$ (Coker 310) significantly surpassed other parents for boll weight, ginning outturn and seed cotton yield, the parents Coker 5114, Dunn 1517 and Lachata had higher means for plant height, number of bolls per plant and lint index, respectively. These four parents showed also significant positive gca effects for the same characters.

Significant desirable gca effects were found in 3 cultivars for plant height, number of bolls per plant, boll weight and ginning outturn, 4 cultivars for lint index, and 2 cultivars for seed cotton yield. The two cultivars Coker 310 and Lachata exhibited significant positive (desirable) gca effects for 5 traits (plant height, number of bolls per plant, boll weight, ginning outturn and

Table (4): Mean performance and sca effects $\left(S_{\text {ih }}\right)$ for different characters for 21 crosses in upland cotton.

\begin{tabular}{|c|c|c|c|c|c|c|c|}
\hline Crosses & & $\begin{array}{l}\text { Plant height } \\
\text { (cm) }\end{array}$ & $\begin{array}{l}\text { No. bolls per } \\
\text { plant }\end{array}$ & $\begin{array}{l}\text { Boll weight } \\
\text { (gm) }\end{array}$ & Lint index (gm) & $\begin{array}{c}\text { Ginning } \\
\text { outturn }(\%)\end{array}$ & $\begin{array}{l}\text { Yield } \\
\text { (gm) }\end{array}$ \\
\hline \multirow{2}{*}{$\mathbf{P}_{1} \mathbf{x} \mathbf{P}_{2}$} & mean & $103.60 \mathrm{~h}$ & $34.07 \mathrm{e}$ & $4.167 \mathrm{~g}$ & $5.600 \mathrm{a}$ & $32.33 \mathrm{i}$ & 141.95 de \\
\hline & $\mathbf{S}_{\mathrm{ih}}$ & $(19.360) *$ & $(2.472)^{*}$ & $(0.015)$ & $(0.449)^{*}$ & $(-1.349) *$ & $(11.071)^{*}$ \\
\hline \multirow{2}{*}{$\mathbf{P}_{1} \times \mathbf{P}_{3}$} & mean & $92.757 \mathrm{n}$ & $27.00 \mathrm{k}$ & 4.433def & $4.567 \mathrm{gh}$ & $34.17 \mathrm{f}$ & $119.72 \mathrm{f}$ \\
\hline & $\mathbf{S}_{\mathrm{ih}}$ & (1.159)* & $(-5.609) *$ & $(0.526)^{*}$ & $(-0.410)^{*}$ & $(0.031)$ & $(-7.066)^{*}$ \\
\hline \multirow{2}{*}{$\mathbf{P}_{1} \times \mathbf{P}_{4}$} & mean & $104.40 \mathrm{~g}$ & $32.43 \mathrm{fg}$ & 4.367d-g & $5.133 \mathrm{~cd}$ & 34.23 ef & $141.63 \mathrm{de}$ \\
\hline & $\mathbf{S}_{\mathrm{ih}}$ & $(10.268)^{*}$ & $(0.524)^{*}$ & $(0.237)^{*}$ & $(0.356)^{*}$ & $(1.381)^{*}$ & $(9.728)^{*}$ \\
\hline \multirow[b]{2}{*}{$\mathbf{P}_{1} \times \mathbf{P}_{5}$} & mean & 81.47 r & $31.03 \mathrm{i}$ & $4.53 \mathrm{de}$ & $5.133 \mathrm{~cd}$ & $31.60 \mathbf{j}$ & $140.68 \mathrm{de}$ \\
\hline & $\mathbf{S}_{\mathrm{ih}}$ & $(-5.458)^{*}$ & $(-2.131)^{*}$ & $(0.256)^{*}$ & $(0.019)$ & $(-1.497)^{*}$ & $(-2.169)$ \\
\hline \multirow{2}{*}{$\mathbf{P}_{1} \times \mathbf{P}_{6}$} & mean & 107.47 e & 33.07 f & $4.267 \mathrm{fg}$ & $5.500 \mathrm{a}$ & 31.63 j & 141.07 de \\
\hline & $S_{\text {ih }}$ & $(20.289) *$ & $(2.069) *$ & $(-0.081)$ & $(0.464)^{*}$ & $(-1.679) *$ & $(7.137)^{*}$ \\
\hline \multirow{2}{*}{$\mathbf{P}_{1} \times \mathbf{P}_{7}$} & mean & 91.07 o & $35.40 \mathrm{~d}$ & 4.600bcd & $3.867 \mathbf{j}$ & 37.17 a & $162.83 \mathrm{c}$ \\
\hline & $\mathbf{S}_{\mathrm{ih}}$ & $(1.149)^{*}$ & $(2.324) *$ & $(0.063)$ & $(-1.088)^{*}$ & $(2.784) *$ & $(12.256)^{*}$ \\
\hline \multirow{2}{*}{$\mathbf{P}_{2} \times \mathbf{P}_{3}$} & mean & $113.20 \mathrm{~b}$ & $27.43 \mathrm{k}$ & $4.167 \mathrm{~g}$ & $4.600 \mathrm{gh}$ & 34.20 ef & $114.31 \mathrm{f}$ \\
\hline & $\mathbf{S}_{\mathrm{ih}}$ & $(15.245)^{*}$ & $(-5.069) *$ & $(-0.085)$ & $(-0.466)^{*}$ & $(-0.375) *$ & $(-22.777)^{*}$ \\
\hline \multirow{2}{*}{$\mathbf{P}_{2} \times \mathbf{P}_{4}$} & mean & $86.33 \mathrm{q}$ & $29.93 \mathrm{j}$ & 4.800 bc & $4.333 \mathrm{i}$ & $34.47 \mathrm{~d}$ & 143.65 de \\
\hline & $\mathbf{S}_{\mathrm{ih}}$ & $(-14.156)^{*}$ & $(-1.876)^{*}$ & $(0.326)^{*}$ & $(-0.532) *$ & $(1.236)^{*}$ & (1.440) \\
\hline \multirow{2}{*}{$\mathbf{P}_{2} \times \mathbf{P}_{5}$} & mean & $101.23 \mathrm{k}$ & $30.87 \mathrm{i}$ & $4.400 \mathrm{~d}-\mathrm{g}$ & 5.200bcd & $36.20 \mathrm{~b}$ & $135.91 \mathrm{e}$ \\
\hline & $\mathbf{S}_{\mathrm{ih}}$ & $(7.952)^{*}$ & $(-2.198)^{*}$ & $(-0.222) *$ & $(-0.003)$ & $(2.725) *$ & $(-17.239 *)$ \\
\hline \multirow{2}{*}{$\mathbf{P}_{2} \times \mathbf{P}_{6}$} & mean & $103.13 \mathrm{i}$ & 29.87 j & $4.833 \mathrm{~b}$ & $5.067 \mathrm{~d}$ & 33.47 h & $144.33 \mathrm{de}$ \\
\hline & $\mathbf{S}_{\mathrm{ih}}$ & $(9.599)^{*}$ & $(-1.031)^{*}$ & $(0.141)^{*}$ & $(-0.058)$ & $(-0.223) *$ & $(0.093)$ \\
\hline \multirow{2}{*}{$\mathbf{P}_{2} \times \mathbf{P}_{7}$} & mean & $92.47 \mathrm{n}$ & 32.07 gh & $5.300 \mathrm{a}$ & $4.867 \mathrm{e}$ & $33.67 \mathrm{~g}$ & $169.96 \mathrm{c}$ \\
\hline & $\mathbf{S}_{\mathrm{ih}}$ & $(-3.808) *$ & $(-0.909) *$ & $(0.419) *$ & $(-0.177)^{*}$ & $(-1.094) *$ & $(9.079) *$ \\
\hline \multirow{2}{*}{$\mathbf{P}_{3} \times \mathbf{P}_{4}$} & mean & 88.33 p & $27.50 \mathrm{k}$ & 4.333efg & $5.300 \mathrm{~b}$ & $33.27 \mathrm{~h}$ & $119.14 \mathrm{f}$ \\
\hline & $\mathbf{S}_{\mathrm{ih}}$ & $(-19.514)^{*}$ & $(-5.317)^{*}$ & $(0.104)^{*}$ & $(0.608)^{*}$ & $(-0.479) *$ & $(-18.973)^{*}$ \\
\hline \multirow{2}{*}{$\mathbf{P}_{3} \times \mathbf{P}_{5}$} & mean & 103.33 hi & 38.13 b & 4.800 bc & $4.500 \mathrm{~h}$ & $33.73 \mathrm{~g}$ & $183.05 \mathrm{~b}$ \\
\hline & $S_{\text {ih }}$ & $(2.693)^{*}$ & $(4.061)^{*}$ & $(0.422)^{*}$ & $(-0.529)^{*}$ & $(-0.256)^{*}$ & $(33.993) *$ \\
\hline \multirow{2}{*}{$\mathbf{P}_{3} \times \mathbf{P}_{6}$} & mean & $102.37 \mathrm{j}$ & $32.43 \mathrm{fg}$ & $4.233 \mathrm{fg}$ & $4.667 \mathrm{fg}$ & $36.23 \mathrm{~b}$ & $137.30 \mathrm{de}$ \\
\hline & $\mathbf{S}_{\mathrm{ih}}$ & $(1.475)^{*}$ & $(0.528)^{*}$ & $(-0.215)^{*}$ & $(-0.284)^{*}$ & $(2.029)^{*}$ & $(-2.841)$ \\
\hline \multirow{2}{*}{$\mathbf{P}_{3} \times \mathbf{P}_{7}$} & mean & $113.40 \mathrm{~b}$ & $31.33 \mathrm{hi}$ & 4.567cde & 5.267 bc & $36.30 \mathrm{~b}$ & 143.09 de \\
\hline & $\mathbf{S}_{\mathrm{ih}}$ & $(9.768)^{*}$ & $(-2.650)^{*}$ & $(-0.070)$ & $(0.397)^{*}$ & $(1.025)^{*}$ & $(-13.695)^{*}$ \\
\hline \multirow{2}{*}{$\mathbf{P}_{4} \times \mathbf{P}_{5}$} & mean & $112.47 \mathrm{c}$ & 43.90 a & 4.367d-g & $4.533 \mathrm{gh}$ & 34.23 ef & $191.62 \mathrm{a}$ \\
\hline & $S_{\text {ih }}$ & $(9.292)^{*}$ & $(10.520) *$ & $(-0.233)^{*}$ & $(-0.295)^{*}$ & $(1.588)^{*}$ & $(37.444) *$ \\
\hline \multirow{2}{*}{$\mathbf{P}_{4} \times \mathbf{P}_{6}$} & mean & 129.17 a & $32.43 \mathrm{fg}$ & $4.467 \mathrm{def}$ & $4.300 \mathrm{i}$ & $33.33 \mathrm{~h}$ & $144.84 \mathrm{~d}$ \\
\hline & $\mathbf{S}_{\mathrm{ih}}$ & $(25.740)^{*}$ & $(1.220)^{*}$ & $(-0.204) *$ & $(-0.451)^{*}$ & $(0.473)^{*}$ & $(-0.418)$ \\
\hline \multirow{2}{*}{$\mathbf{P}_{4} \times \mathbf{P}_{7}$} & mean & $97.30 \mathrm{I}$ & $33.27 \mathrm{f}$ & 5.067 a & $4.833 \mathrm{e}$ & $33.33 \mathrm{~h}$ & $168.55 \mathrm{c}$ \\
\hline & $\mathbf{S}_{\mathrm{ih}}$ & $(-8.867)^{*}$ & $(-0.024)$ & $(0.207)^{*}$ & $(0.164) *$ & $(-0.597) *$ & $(6.639) *$ \\
\hline \multirow{2}{*}{$\mathbf{P}_{5} \times \mathbf{P}_{6}$} & mean & $106.63 \mathrm{f}$ & 31.57 hi & $5.300 \mathrm{a}$ & 4.767 ef & $35.33 \mathrm{c}$ & $167.31 \mathrm{c}$ \\
\hline & $\mathbf{S}_{\mathrm{ih}}$ & $(10.415)^{*}$ & $(-0.902) *$ & $(0.481)^{*}$ & $(-0.321)^{*}$ & $(2.229)^{*}$ & $(11.099)^{*}$ \\
\hline \multirow{2}{*}{$\mathbf{P}_{5} \times \mathbf{P}_{7}$} & mean & $109.33 \mathrm{~d}$ & $38.27 \mathrm{~b}$ & $5.133 \mathrm{a}$ & $5.200 \mathrm{bcd}$ & $34.50 \mathrm{~d}$ & 196.43 a \\
\hline & $\mathbf{S}_{\mathrm{ih}}$ & $(10.374) *$ & $(3.720)^{*}$ & $(0.126)^{*}$ & $(0.194)^{*}$ & $(0.325)^{*}$ & $(23.579) *$ \\
\hline \multirow{2}{*}{$\mathbf{P}_{6} \times \mathbf{P}_{7}$} & mean & $93.50 \mathrm{~m}$ & $36.30 \mathrm{c}$ & 4.467def & $4.867 \mathrm{e}$ & 34.40 de & $162.14 \mathrm{c}$ \\
\hline & $\mathbf{S}_{\mathrm{ih}}$ & $(-5.711)^{*}$ & $(3.920) *$ & $(-0.611)^{*}$ & $(-0.062)$ & $(0.010)$ & $(-1.799)$ \\
\hline \multicolumn{2}{|c|}{ Crosses means } & 101.569 & 32.776 & 4.600 & 4.862 & 34.181 & 150.929 \\
\hline \multicolumn{2}{|c|}{ SE (Sij) } & 0.249 & 0.412 & 0.099 & 0.064 & 0.099 & 3.551 \\
\hline
\end{tabular}

$P_{1}=$ Halab 90, $P_{2}=$ SP 8886, $P_{3}=$ Dunn 1517, $P_{4}=$ Coker 5114, $P_{5}=$ Lachata, $P_{6}=$ Dunn 1047 and $P_{7}=$ Coker 310 
seed cotton yield) and 4 traits (number of bolls per plant, boll weight, lint index and seed cotton yield) characters respectively. These could be used as donor parents for the above mentioned characters in hybridization programmes. Other cultivars exhibited good gca for 1 to 3 characters and could be used for improvement of these characters. Hassan et al. (2000), Ahmad et al. (2005) and Abro et al. (2009) reported that the best performance parents with high gca produce the best hybrid combinations.

The sca effects and means of the crosses for different characters are presented in Table 4. The crosses $\mathrm{P}_{4} \times \mathrm{P}_{6}, \mathrm{P}_{4} \times \mathrm{P}_{5}, \mathrm{P}_{5} \times \mathrm{P}_{6}, \mathrm{P}_{1} \times \mathrm{P}_{2}, \mathrm{P}_{1} \times \mathrm{P}_{7}$ and $\mathrm{P}_{5} \times \mathrm{P}_{7}$ showed significantly higher mean performance for plant height, number of bolls per plant, boll weight, lint index, ginning outturn and seed cotton yield, respectively, and at the same time they have positive desirable mid parents heterosis, and also five of them (excluding $\mathrm{P}_{1} \mathrm{x}$ $\mathrm{P}_{2}$ ) showed positive desirable heterosis over higher parent. The crosses $\mathrm{P}_{1} \times \mathrm{P}_{4}$ and $\mathrm{P}_{5} \times \mathrm{P}_{7}$ showed significantly positive sca effects for all the studied characters. Six crosses viz, $\mathrm{P}_{1} \times \mathrm{P}_{2}$ and $\mathrm{P}_{1} \times \mathrm{P}_{6}$ (for lint index), $\mathrm{P}_{3} \times \mathrm{P}_{5}$ (for number of bolls per plant), $\mathrm{P}_{3} \times \mathrm{P}_{7}$ (for number of bolls per plant and ginning outturn), $\mathrm{P}_{5} \times \mathrm{P}_{6}$ (for number of bolls per plant) and $\mathrm{P}_{5} \times \mathrm{P}_{7}$ (for number of bolls per plant and seed cotton yield) involved high $\mathrm{x}$ high interaction of gca of the parents, while some other crosses involved low $\mathrm{x}$ high or low $\mathrm{x}$ low interactions.

The crosses $\mathrm{P}_{1} \times \mathrm{P}_{7}, \mathrm{P}_{2} \times \mathrm{P}_{5}$ and $\mathrm{P}_{6} \times \mathrm{P}_{7}$, although showing high mean performance for boll weight, lint index seed cotton yield, respectively, did not show significant or desirable sca effect. This indicats that high mean performance value per se of the cross may not necessarily indicate their potentiality in crosses. Moll and Stuber (1974) reported that any combination among the parents may produce higher hybrid vigor over parents, which might be due to dominant, over dominant or epistatic gene action. So, the crosses showing desirable sca effects can be used in future breeding programme.

It was concluded that cultivars $\mathrm{P}_{5}$ (Lachata) and $\mathrm{P}_{7}$ (Coker 310) being good general combiners for most of the characters (number of bolls per plant, boll weight, lint index and seed cotton yield) and (plant height, boll weight, ginning outturn and seed cotton yield), respectively, can be considered good breeding materials to be exploited in breeding programs for the improvement of these characters, while the crosses $\mathrm{P}_{1} \times \mathrm{P}_{4}$ (Halab $90 \times$ Cocker 5114) and

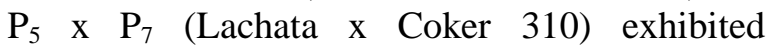
significant desirable specific combining ability for all studied characters. However, the crosses $\mathrm{P}_{1} \times \mathrm{P}_{4}$ (Halab $90 \times$ Cocker 5114), followed by $\mathrm{P}_{5} \times \mathrm{P}_{7}$ (Lachata $\mathrm{x}$ Coker 310) exhibited high positive heterosis over mid parents and higher parent for most of the studied characters, and could be exploited in producing hybrid cotton.

\section{REFERENCES}

Abro S., Kandhro M. M., Laghari S., Arain M. A. and Deho Z. A. (2009). Combining ability and heterosis for yield contributing traits in upland cotton (G. hirsutum L.). Pak. J. Bot., 41(4): 1769-1774.

Ahmad R. D., Malik A. J., Hassan G. and Subhan M. (2005). Estimation of combining ability for seed cotton yield and its components in inter-varietal crosses of cotton. Camal Univ. J. of Res., 21: 1-6.

Altaf M., Sadaqat H. A., Khan M.A. and Ubair M. Z. (1996). Genetic variability and correlation studies in some promising cotton strains under cotton leaf curl virus epidemic. The Pak. Cottons, 40(3- 4): 8090.

Basal T. and Turgut I. (2003). Heterosis and combining ability for yield components and fiber quality parameters in half diallel cotton (G. hirsutum L.). Turk. J. Agric., 27: 207-212.

Deshpande L. A and Baig K. S. (2003). Combining ability analysis for yield, economic and morphological traits in American cotton (Gossypium hirsutum L.). Journal of Research, ANGRAU, 31(3): 28-34.

Griffing J.B. (1956). Concept of general and specific combining ability in relation to diallel crossing system. Austral. J. Biol. Sci., 9: 463-493.

Halluer A. R. and Miranda J. B. (1981). Quantitative genetics in maize breeding. Iowa State Univ. Press, Ames, USA.

Hassan G., Mahmood G., Razzaq A. and Hayatullah (2000). Combining ability in inter-varietal crosses of upland cotton. Sarhad J. Agri., 16(4): 407-410.

Hayman B.I. (1954). The theory and analysis of diallel crosses. Biometrics, 10: 235-244.

Hayman B.I. (1960). The theory and analysis of diallel crosses III. Genetics, 45: 155-172. 
Inamullah H. A., Muhammad F., Sirajuddin G. Hassan G. and Gul R. (2006). Evaluation of heterotic and heterobeltiotic potential of wheat genotype for improved yield. Pak. J. Bot., 38(4): 1159-1168.

Jinks J. L. (1956). The $F_{2}$ and backcross generation from a set of diallel crosses. Heredity 10: 1-30.

Jinks J. L. and Hayman B. I. (1953). The analysis of diallel crosses. Maize Genet. Coop. News Letter, 27: 48-54.

Khan N. U., Abro H. K., Kumbhar M. B., Hassan G. and Khan M. (1999). Study of heterosis in upland cotton - 1. Quality traits. The Pak. Cotton, 43(3-4): 53-64.

Moll R. H. and Stuber C.W. (1974). Quantitative genetics empirical results relevant to plant breeding. Ad. Agron. J., 26: 277-313.

Mukhtar M. S. and Khan T. M. (2000). Estimation of heterosis for yield and its components in cotton. Pak. J. Bio. Sci., 3: 1412-1413.

Naquibullah H., Abro K., Lumbhar M. B., Hassan G. and Mahmood G. (2000). Study of heterosis in upland cotton-II.
Morphology and yield traits. The Pak. Cottons, 44 (1-2): 13-23.

Salam C. A. (1991). Status of itnra-specific commercial hybrids in Punjab. The Pak. Cottons, 35 (1): 5-17.

Solangi M., Baloch H., Bhutto H., Lakho A. R. and Solangi M. H. (2002). Hybrid vigour in intraspecific crosses of $\mathrm{F} 1$ hybrids of $G$. hirsutum $\mathrm{x}$ G. barbadense. Pak. J. Bio. Sci., 4: 945- 948.

Sprague G. F. and Tatum L. A. (1942). General vs specific combining ability in single crosses of corn. J. American Soc. Agron., 34: 923-52.

Steel R. G. D. and Torrie J. H. (1980). Principles and Procedures of Statistics. ( $2^{\text {nd }}$ ed.). McGraw Hill Inc., New York.

Verhalen L. M. and Murray J. C. (1967). A diallel analysis of several fiber properties traits in upland cotton (G. hirsutum L.). Crop. Sci. 7: 501-505.

Zia-ul-Islam H., Sadaquat A. and Khan F.A. (2001). Combining ability of some hirsutum cotton types for economic traits. International J. of Agric. and Biol., 3 (4): 411-412.

$$
\begin{aligned}
& \text { قوة الهجين والقرة على الايتلاف في الهجن التبادلية بين أصناف من القطن الابلند }
\end{aligned}
$$

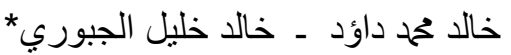

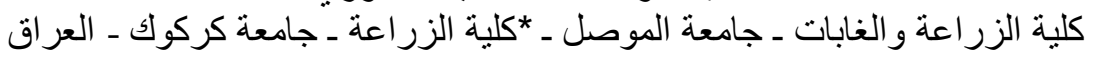

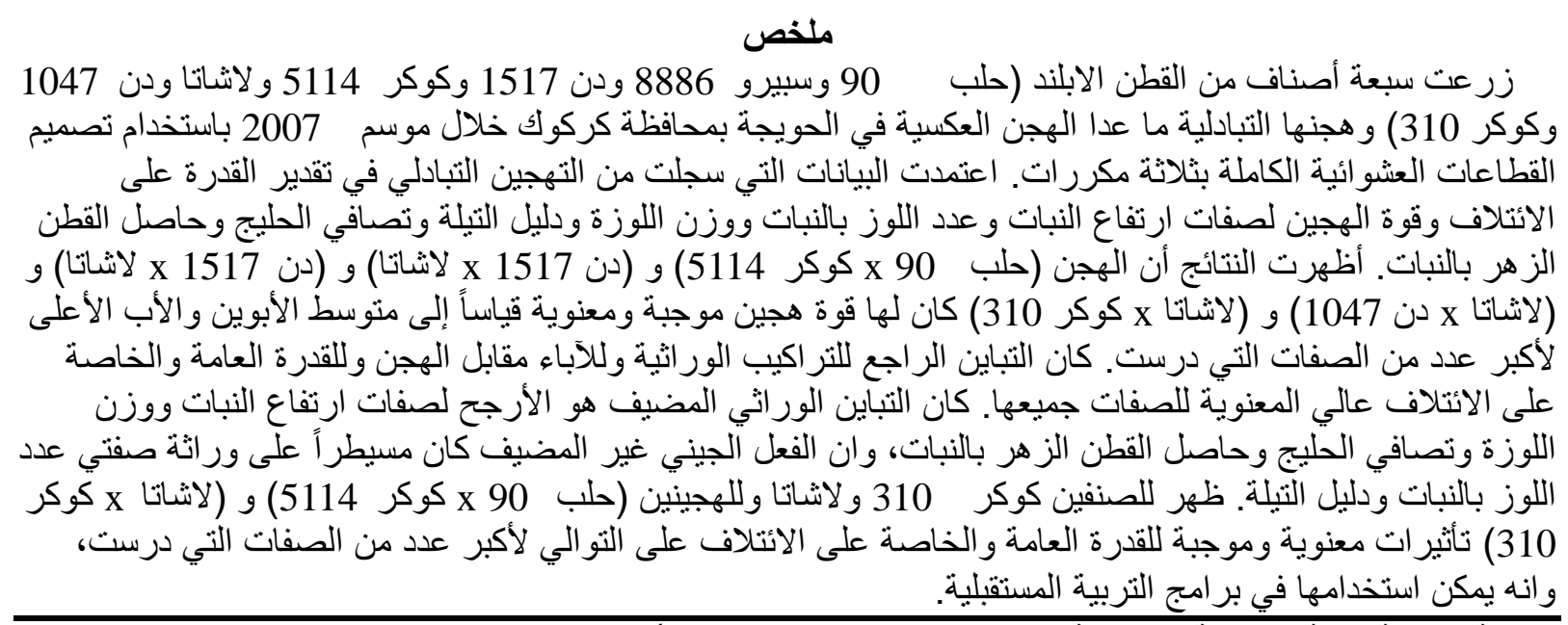

المجلة العلمية لكلية الزراعة - جامعة القاهرة - المجلد (61) العدد الأول ( يناير 2010):1:-10. 